\title{
Human motion behavior while interacting with an industrial robot
}

\author{
Dino Bortot ${ }^{\mathrm{a},}$, , Hao Ding ${ }^{\mathrm{b}}$, Alexandros Antonopolous ${ }^{\mathrm{a}}$ and Klaus Bengler ${ }^{\mathrm{a}}$ \\ ${ }^{a}$ Institute of Ergonomics, Technische Universität München, Boltzmannstraße 15, 85747 Garching bei München, \\ Germany \\ ${ }^{\mathrm{b}}$ Control and System Theory, University of Kassel, Wilhelmshoeher Allee 73, 34121 Kassel, Germany
}

\begin{abstract}
Human workers and industrial robots both have specific strengths within industrial production. Advantageously they complement each other perfectly, which leads to the development of human-robot interaction (HRI) applications. Bringing humans and robots together in the same workspace may lead to potential collisions. The avoidance of such is a central safety requirement. It can be realized with sundry sensor systems, all of them decelerating the robot when the distance to the human decreases alarmingly and applying the emergency stop, when the distance becomes too small. As a consequence, the efficiency of the overall systems suffers, because the robot has high idle times. Optimized path planning algorithms have to be developed to avoid that. The following study investigates human motion behavior in the proximity of an industrial robot. Three different kinds of encounters between the two entities under three robot speed levels are prompted. A motion tracking system is used to capture the motions. Results show, that humans keep an average distance of about $0,5 \mathrm{~m}$ to the robot, when the encounter occurs. Approximation of the workbenches is influenced by the robot in ten of 15 cases. Furthermore, an increase of participants' walking velocity with higher robot velocities is observed.
\end{abstract}

Keywords: human-robot interaction, human-robot coexistence, human motion behavior, safety distance

\section{Introduction}

\subsection{HRI as a proper solution to ergonomic problems}

Current issues in industrial production call for the development of HRI applications.

Industrialized countries are faced with demographic changes among the work force, including a significant decrease in workers' physical abilities. Production planning must create new strategies to combat this problem. The reinforcement of strategies to rationalize work and its organization prevents any reduction in the number of workers subjected to strenuous constraints and maintains aging workers in situations in which these constraints persist [2]. Additionally, ergonomists will still face critical processes/situations where the classic ergonomic workplace design is not sufficient to generate healthy working conditions. The concept of HRI seems to be a suitable solution to these problems.
Regarding the development process, ergonomists must understand how safe, ergonomic and accepted systems can be developed while increasing efficiency. If humans are allowed to work in the same workspace as industrial robots, potential collisions may occur. The avoidance of such and, therefore, the minimization of dangerous and hazardous impacts, have to be guaranteed by the safety system. On the basis of calculated distances between the two interacting entities, the robot can be controlled accordingly (e.g. lowering its velocity or changing its direction). In doing this, the system's overall efficiency may suffer as those control actions always imply a system status with lower productivity [10]. Therefore, it is crucial to avoid collisions that would trigger a safety reaction [12]. Up to now not realistic human motions, respectively, human behavior that has traceable low probabilities of occurrence, are considered. Risk assessments cover every possible misbehavior or malfunction $[4,5]$. This leads to complex system archi-

\footnotetext{
* Corresponding author. E-mail: bortot@tum.de. Phone: +49 89289 15383. Fax: +49 8928915389
} 
tectures, expensive sensor systems on the one hand and unwanted system performance (low productivity) on the grounds of safety issues on the other hand. If information about upcoming human behavior would be considered in the robot's path planning, subsequent possible collisions could be avoided in an early phase and the system's performance would increase.

\subsection{Previous ergonomic investigations in HRI}

The first studies dealing with human perception/awareness of industrial robots were conducted by Nagamachi and Anayama [[7] cit. in [6]] in 1983. Students were asked to position themselves as close to a robot as they felt safe. From the author's point of view participants approximated the robot too close: the average distance at the lowest of five robot velocity levels $(0,14 \mathrm{~m} / \mathrm{s})$ was $1,5 \mathrm{~cm}$. When the robot moved faster $(0,46 \mathrm{~m} / \mathrm{s})$ the average distance increased to $19,5 \mathrm{~cm}$.

To review Parsons' idea to sensitize workers for safety-conscious behavior around industrial robots by simulating an accident [9], Karwowski et al. examined the effect of simulated accidents on human perception within HRI [6]. They performed an independent samples study, one where an accident/collision was experienced before the actual experiment, and the other one not. The study showed the significant effect of a simulated robot accident on safety behavior of industrial workers. Workers that were exposed to an accident were more careful in that they intruded less frequently into the robot's work envelope than did the workers who did not witness the accident [6].

Various studies were conducted that comment on the influence of the robot's velocity on human perception. Shibata and Inooka investigated different robot motions on their human-like appearance [11]. They used seven adjective/word pairs to evaluate the human-likeness. As a result, they stated that humanlikeness to human arm motion and robot motion depends on the velocity peak position in the movement time. That is, it is clarified that one of the conditions which makes humans perceive a motion to be human-like is that the velocity peak position locates at the appropriate front position in movement time [11]. Furthermore the results reveal that there exist appropriate maximum velocity values which make humans perceive a motion to be the most human-like, and humans begin to assess motions as inhuman-like if the maximum velocity values of the motions exceed the above appropriate values [11].
Yamamoto et al. analyzed human emotions against motions of an industrial robot with two degrees of freedom [16]. Therefore, they placed participants of the study in front of the robot and asked them to follow a given reference path to interfere with the robot. The robot's arm approximated the human under different velocities (four levels between $0,1 \mathrm{~m} / \mathrm{s}$ and $0,4 \mathrm{~m} / \mathrm{s}$ ). Having reached a certain distance, the robot began to follow an optimized path, avoiding the human hand in a constant distance $(0,1 \mathrm{~m} ; 0,15 \mathrm{~m} ; 0,2 \mathrm{~m}$; and $0,25 \mathrm{~m})$. The results show that the emotion of "pleasant" is representative, that is, improving this emotion will make all the other emotions better. For the optimal velocity, the authors come to the conclusion that $0,2 \mathrm{~m} / \mathrm{s}$ are perceived the optimum.

The question whether the distance between human and robot, the velocity of the robot, or interference of the two variables, have the deepest impact on human perception, is not answered consistently. Tejima [13] and Hanajima [3] figured out, that the distance between the two entities has the biggest influence on human perception. On the other hand an interaction between the distance and the velocity was discovered as a result of the study of Yamada et al. [15].

All of the above mentioned studies deal with human perception of industrial robot motions. Designing human-robot interactions in such a way that human users are not scared or afraid is an important requirement to guarantee user acceptance. However, beyond that, the system's efficiency has to be considered. Because of this the following study, investigating human motion behavior to increase efficiency of human-robot interaction by ensuring smooth collaboration, has been conducted.

\section{Method}

\subsection{Framework conditions of the study}

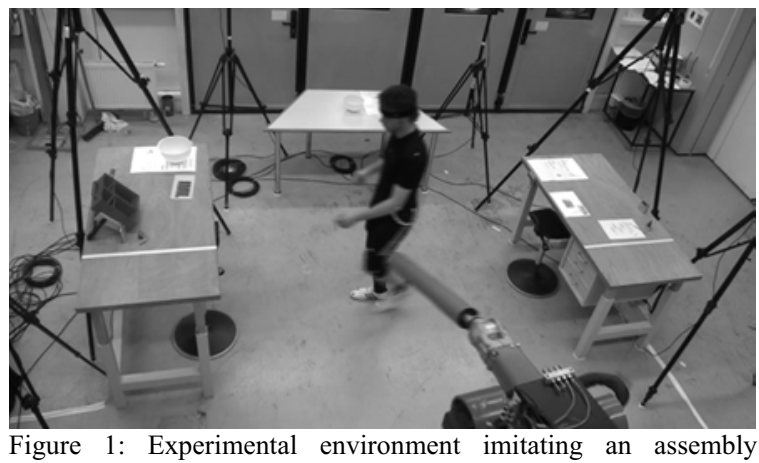

Figure 1: Experimental environment imitating an assembly workstation 
The setup of the study (within subject design) embodied HRI in an assembly environment (Fig. 1). Participants moved between three workbenches. Parts were provided on and collected from the workbenches by an (one-arm) industrial robot that reaches temporarily into the working space of the human. As a consequence, collisions between human and robot were possible.

The study was conducted with 15 participants with an average age of 26,2 (tandard deviation $\mathrm{SD}=3,2$ ). Twelve of them were male, three female. They all indicated at least an above-average trust in technical systems and had no or only little previous experience with industrial robots (Fig. 2).

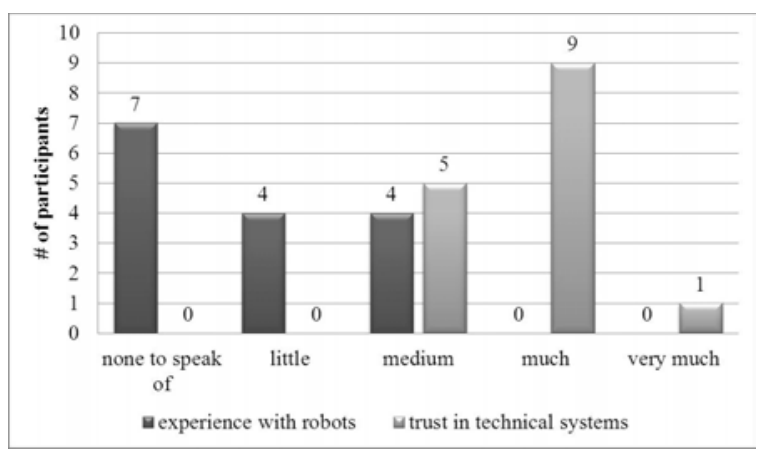

Figure 2: Characterization of the subject group

\subsection{Safety issues}

The conflict to ensure safety at all times but also warrant a realistic reflection of a typical industrial environment had to be solved. Since no safety system, for example a camera-based surveillance of the human and the robot, had yet been installed, the robot was operated manually by the experimenter controlling the dead-man's button. Nevertheless, collisions between the two interaction partners (human/robot) still were feasible. Therefore a dummy based on foam material was magnetically attached at the robot's tool center point (TCP) (Fig. 3). In case of a collision the actual impact on the human would be diminished for two reasons: on the one hand the dummy would drop, as the effective magnetic forces were chosen accordingly; on the other hand, the soft foam material surrounding the dummy would have a softening effect on the bounce. Thereby human-safety was ensured and the two interaction partners could safely share a common workspace like in future HRI scenarios.

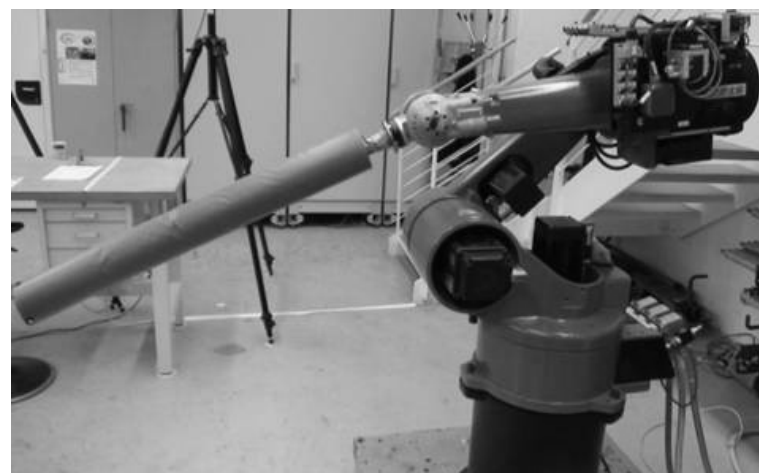

Figure 3: Magnetic linkage between the foam dummy and the TCP

\subsection{Procedure}

Participants were asked to perform tasks on workbenches. The two main workstations (left and right in Fig. 1) afforded the opportunity to work in a standing position on the left respectively in a sitting position on the right (and vice versa). Between tasks, participants had to move from one bench to another. The robot moved on predefined trajectories during the fulfillment of tasks, and in particular as the human crossed the experimental field to go to the other workbench. As a consequence, encounters 'human vs. robot' occurred (Fig. 4). Each participant completed six runs under three different robot velocity levels, each of them provoking eight encounters.

\subsubsection{Human and robot (motion) behavior}

Due to the experimental design humans' and robot's trajectories could intersect in different ways. Fig. 4 shows three varying encounters that were provoked during the experiment.

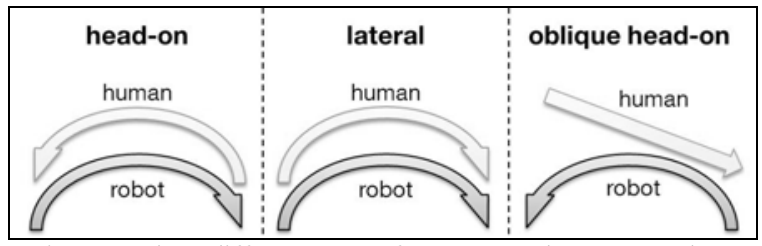

Figure 4: Three different types of encounters 'human vs. robot'

Head-on encounters occurred five times per run, as the human walked from one side to the other and countered the robot that was coming from the other side. Lateral encounters (human and robot going simultaneously from one side to the other) happened twice and the oblique head-on encounter (human coming from the table above (Fig. 1) facing the robot that came from the right workbench) once. Taken into account six runs each participant encountered the 
robot 48 times. To investigate changes in human motion behavior depending on the velocity of the robot three different velocity levels were applied, each of them two times. Within the second run the premises for the work posture were changed. To investigate effects of learning robot velocity was changed in each case beginning with level 1 , following up by level 2 and ending with level 3 (Table 1).

Table 1

Average and maximum velocities of the robot $[\mathrm{m} / \mathrm{s}]$

\begin{tabular}{|l|c|c|}
\hline & average speed & maximum speed \\
\hline level 1 & 0,55 & 1,41 \\
\hline level 2 & 0,76 & 2,14 \\
\hline level 3 & 0,88 & 2,83 \\
\hline
\end{tabular}

Table 1 shows the applied velocity levels of the robot. Level 1 represents 20\% PTP- (point to point), level $2 \quad 30 \% \quad$ PTP- and level $3 \quad 40 \%$ PTP-velocity. This led to different average and maximum values, as the PTP-mode realizes different accelerating and decelerating profiles depending on distance and direction of the TCP.

The three levels were chosen based on the safety issues, whereby the highest value embodied the highest possible without seriously endangering participants. Robot motions considered for this calculation were not exhaustive. Those, happening while the human fulfilled a task at a workbench were ignored and just the motions that lead to encounters were attended.

\subsubsection{Tasks}

On the workbenches different tasks had to be completed. All of them followed realistic production scenarios, i.e. required manual skills and/or cognitive capacity of the participants. One group of tasks demanded to sort different-colored LEGO bricks and to then use them to build the emblem of the university. The second group required thinking and calculating skills in solving simple arithmetic problems. Even though the focus was on human motion behavior encountering a moving robot, the participants were pushed for time during the assignments and got the instruction to complete the tasks as quickly and accurately as possible. They were consciously led astray; to that effect participants tried their best in solving the tasks on the workbenches and did not concentrate on the motion behavior while they encountered the robot. In doing this, the supervisors of the study anticipated to bring about the humans' most natural motion behavior.

\subsection{Apparatus}

The captured data consisted of objective key figures to analyze and describe human motion behavior as well as questionnaires to describe the subjectively felt stress.

\subsubsection{Objective key figures}

The optoelectronic motion capturing system Vicon [14] captured the movements of both partners through reflecting markers which were placed on designated fulcrums of the human and on the robot. All together 34 markers were placed on the two interaction partners (31 on the human, 3 on the TCP (of the dummy)) (Fig. 5; above, left).

Most of the markers were placed as redundant ones to ensure that lost data could be replaced. Subsequently, only a few markers were used for the analysis of the experiment.

Next to the infrared cameras of the Vicon system a digital camera was used to assess the participants' sitting-down- and standing-up-behavior. It was placed two meters above the experimental area Fig. 1 is an exemplary screenshot from the camera's point of view.

\subsubsection{Subjective ratings}

To measure the subjective workload of the participants and their felt strain, they filled out the NASA TLX evaluation sheet [8]. Secondly, they answered a self-made survey after the experiment.

\subsection{Variables to describe human motion behavior}

The following factors are identified as appropriate factors to analyze and describe human motion behavior:

- trajectories of human motions in the course of encounters with the robot

- distance between human and the robot (TCP and robot base) during the encounters

- strategy in approximating the workbenches

- operating human walking velocity

\section{Results}

\subsection{Human trajectories encountering the robot}

Fig. 5 exemplary illustrates the trajectories for each encounter type. 


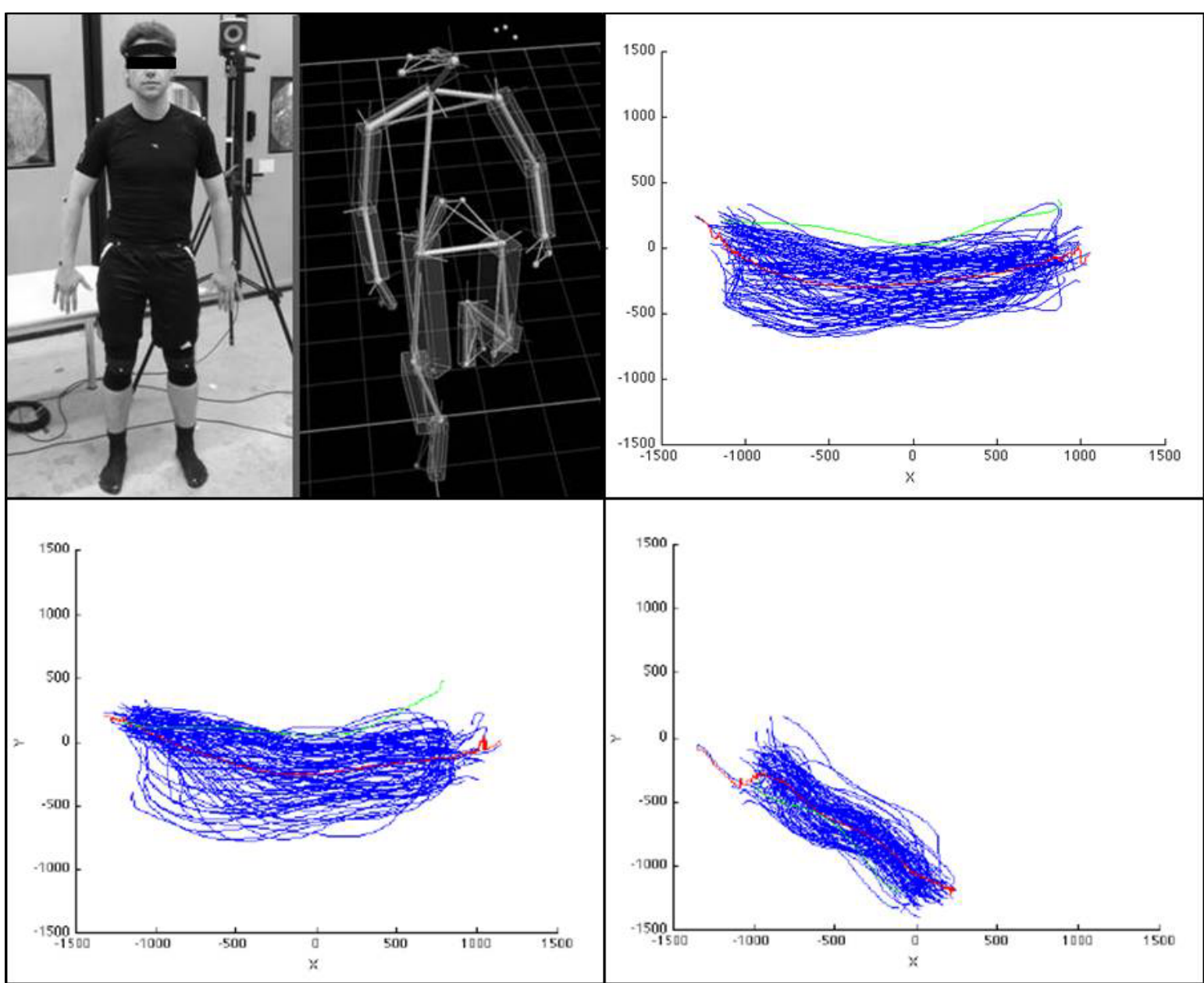

Figure 5: Data of the Vicon system (above, left) is processed in MATLAB. Trajectory clusters show human motion behavior [mm].

The data was originated from the marker that was placed in the neck of the participants. Due to its central location and its visibility at any times of the experiment, this marker was suitable the best. Fig. 5 depicts all of the 15 participants' trajectories for the three different encounter kinds. For the first two types (head-on (Fig. 5; above, right) and lateral (Fig. 5; below, left)), arc-shaped trajectories can be observed. Generally, big lateral deviations among the participants appear (up to $1 \mathrm{~m}$ ), which could be due to participants' individual senses of safety. Nevertheless, a comparison to the baseline (captured trajectory with no robot interference) shows a generally changed motion pattern of the participants. For the head-on encounter this difference is a little smaller than for the lateral ones.

Oblique head-on encounters (Fig. 5; below, right) lead to S-shaped trajectories. Walking from the starting point to their destination people choose the direct connection. Encountering the robot (coming from the rear left side) they change their motion pattern to a left direction, as the robot continues its motion to the right. After the robot has passed, they finish their motion going directly to the point of destiny. The difference between the average and the baseline trajectory is smaller than the one in the other two cases.

\subsection{Minimal distance between the two entities}

The distance to the robot kept by the humans is one possible factor to describe human motion behavior in HRI scenarios. This study looks at two different distances.

\subsubsection{Distance human to robot base}

The analytical identification of the distance human to robot base has been done only for the head-on and the lateral encounters. It was measured right in the middle of the path from one workbench to the other. Table 2 shows the results of the measurement. 
Table 2:

Average distance human to robot base in the middle of the crossing motion $[\mathrm{cm}]$

\begin{tabular}{|c|c|c|}
\hline encounter & $\begin{array}{c}\text { velocity of the } \\
\text { robot }\end{array}$ & $\begin{array}{c}\text { average dis- } \\
\text { tance }[\mathrm{cm}]\end{array}$ \\
\hline \multirow{4}{*}{ head-on } & level 1 & 166,01 \\
\hline & level 2 & 167,00 \\
\hline & level 3 & 168,53 \\
\hline & overall & 167,18 \\
\hline \multirow{4}{*}{ lateral } & level 1 & 175,10 \\
\hline & level 2 & 181,76 \\
\hline & level 3 & 180,18 \\
\hline & overall & 179,01 \\
\hline
\end{tabular}

The average distance of the lateral encounters is bigger than the one of the head on ones. A reason for that is the fact that, at times, the robot moved in the humans' back, which causes feelings of insecurity.

For the head-on encounters a small growth in distance depending on robot velocity can be observed. This cannot be approved for the lateral encounters. A closer look at the standard deviation reveals that head-on encounters are, in contrast to lateral ones, smaller and performed more similar among the participants (Table 3).

Table 3

Standard deviation intervals of the distance for head-on and lateral encounters in $[\mathrm{cm}]$

\begin{tabular}{|l|c|c|}
\hline encounter & head-on & lateral \\
\hline level 1-3 & {$[17,28 ; 23,88]$} & {$[23,10 ; 40,31]$} \\
\hline
\end{tabular}

\subsubsection{Distance human to TCP}

To determine each human's closest approximation to the TCP the following analysis process is applied. First, the temporary distance between all markers and the TCP is determined. After that, the value of the closest marker (shortest distance at time $t$ ) is saved. Fig. 6 shows the results of the average minimal distance depending on robot velocity and type of encounter.

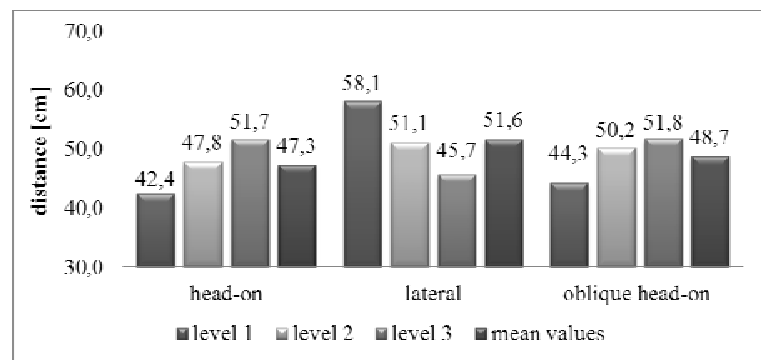

Figure 6: Average minimal distance human to TCP in [cm]
Generally, larger average distances during lateral encounters (mean value: $51,6 \mathrm{~cm}$ ) compared to headon (mean value: $47,3 \mathrm{~cm}$ ) and oblique head-on encounters (mean value: $48,7 \mathrm{~cm}$ ) can be observed. This could be justified with the participants' limited ability to assess the robot's motions in their back. Because of this, humans would feel more insecure and choose a larger distance.

With an incrementing velocity of the robot the minimal distance during head-on and oblique head-on encounters increases. This cannot be approved for lateral encounters. No certain pattern can be detected.

The smallest distance of one single person altogether is beneath $5 \mathrm{~cm}$ (Fig. 7). Taking into account robot velocities up to $2 \mathrm{~m} / \mathrm{s}$ and more it has to be scrutinized, whether this approximation can be justified with reasonable motion planning next to the robot. It could be due to learning effects, since robot trajectories did not change in the course of the experiment, but were only performed with higher velocities. It is conceivable that participants recognized that the robot will not change its motion patterns and therefore, even under conditions with higher robot speed, approximate it to a very small distance.

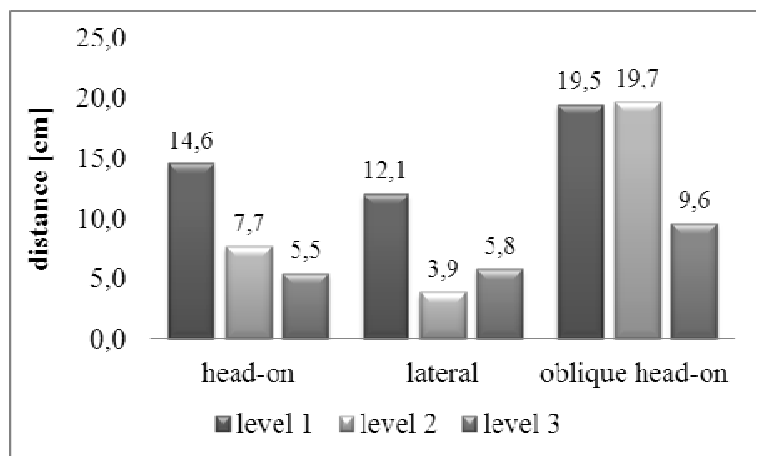

Figure 7: Overall smallest distance of participant number to the TCP in $[\mathrm{cm}]$

To define safety regions, i.e. zones in which the robot can move without endangering the human, information about the occupied space by the human is required. Therefore, it is important to look at the differences among the participants; the bigger they are, the larger the safety region has to be defined, and, as a consequence, the lower productivity of the overall system will be. Table 4 sums up minimum, average and maximum values, as well as the standard deviation for the smallest distance chosen by single participants for all three velocity levels. 
Table 4

Extreme, average and standard deviation values for the smallest distance to the TCP in $[\mathrm{cm}]$ chosen by single participants

\begin{tabular}{|l|c|c|c|c|}
\hline & min & max & avg & SD \\
\hline head-on & 30 & 69 & 47,3 & 12,5 \\
\hline lateral & 31 & 69 & 51,6 & 12,3 \\
\hline $\begin{array}{l}\text { oblique } \\
\text { head-on }\end{array}$ & 30 & 71 & 48,7 & 13,3 \\
\hline
\end{tabular}

All three types are characterized by similar average, minimum and maximum values. That means that safety regions with low safety requirements (that consider only average values) could be defined quite well. If a higher safety standard has to be applied, the minimum and maximum values have to be considered. With differences in the region of $40 \mathrm{~cm}$ the forbidden region for the robot increases significantly and productivity suffers.

\subsection{Sitting down and standing up}

As described before, participants had to sit down at the workbenches to complete the tasks and later on stand up again after they were done with the job. Doing this noticeably many of them used a wellthought strategy to avoid collision with the robot. The data of the digital camera was used to detect different strategies. Two main categories could be distinguished: strategies depending on the robot and such, not depending on the robot. For both of them two different varieties exist (Fig. 8).

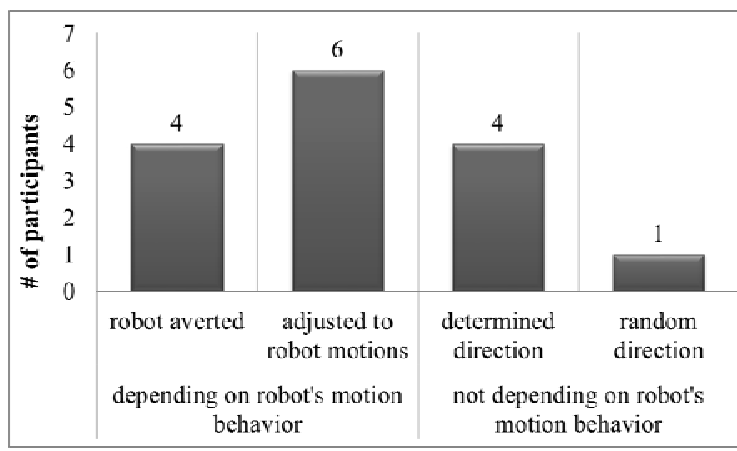

Figure 8: Participants' sitting down and standing up behavior

Robot averted strategy means that the proband always approached and left the workbench on this side of the chair, where collisions with the robot were hardly probable. If the participant adjusted his behavior after maximal two encounters, such that previous random approximations are replaced by planned ones on the outer side of the chair, they are classed as motions adjusted to the robot. Two thirds of the participants used either of the two described strategies, i.e. a strategy depending on the robot. The others either had a fixed direction approaching the workbenches that did not depend on the robot (four participants) or did not follow any strategy at all (one case).

Further investigation of the data proved that strategies did not change under different circumstances, i.e. different robot velocities.

\subsection{Human walking velocity}

Looking at human walking velocity during the encounters, a further effect appeared. If different robot velocity levels were applied, human walking velocity changed. It was measured with the marker in the neck of the proband, as it clearly indicates walking velocity of the overall body and not just one single limb. Fig. 9 illustrates the fact that the more human walking velocity increases the faster the robot moves.

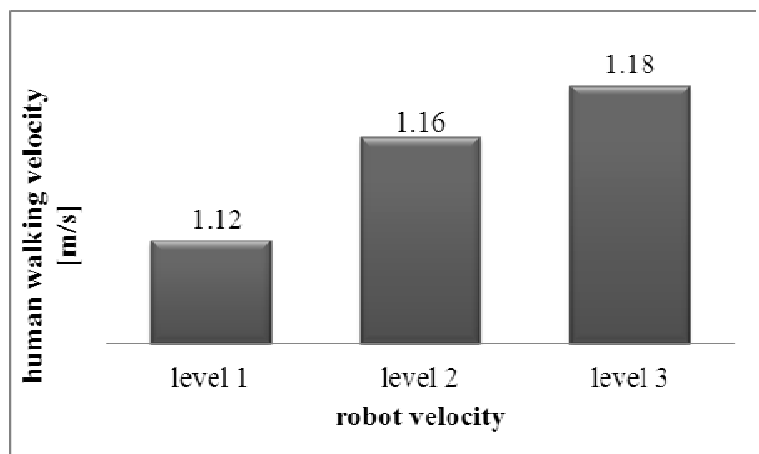

Figure 9: Human walking velocity as a function of robot speed

\subsection{Results of the questionnaires}

Three participants mentioned a sense of insecurity during their interaction with the robot, which was confirmed by the results of the NASA TLX survey. Such participants that felt secure at all times quantify their subjective stress with an average NASA TLX index of 0,34. Comparatively, the index of the three mentioned persons is distinctly higher: 0,49 .

However, only $20 \%$ of participants indicate a significant influence of the robot on their (motion) behavior. 


\section{Discussion}

No general valid description of human motion behavior depending on robot velocity can be given. Most of the participants felt safe at all times of the experiment (result of the concluding survey). Major changes in human motion behavior due to felt anxiety cannot, at least not for the minimal distances, be determined. This, indeed, is surprising, as the maximum robot speed in the experiment was up to ten times the velocity of the ISO-standard. A possible reason for that could be that all participants experienced robot velocities in the same order (level 1, level 2, and level 3), i.e. did already know the motions of the robot and, because of that, risked very small distances to the TCP, even though velocity increased. As long as robot behavior does not change, higher velocities do not seem to be a problem.

The overall trust in the system and the close approximation to the robot could result from the fact that the TCP actually was not equipped with a sharp tool or the like, but conveyed the impression of safety due to the foam dummy. It must be assumed that the approximation would enlarge, if actual tools on the TCP would handle parts from one point to the other.

The fact that the study is conducted with young, inexperienced participants who trust technical systems has to be kept in mind. It must be assumed that operators in production halls might have a negative mindset towards industrial robots as their attention was continuously drawn to the hazards that arise from such machines. Thus, human motion behavior of participants that work in industrial production should be more cautious than that of the investigated participants.

\section{Summary and outlook}

A study with 15 participants investigating human motion behavior in the proximity of an industrial robot has been presented. Three different kinds of encounters between the two entities under three robot velocity levels were implemented. A Vicon system was used to capture the motions. Results showed arcshaped trajectories around the robot and an average distance of about $0,5 \mathrm{~m}$ between the two entities, when the encounter occurred. $80 \%$ of the participants did not feel insecure during the experiment, which seems to be retraceable to constant, non-adaptive robot behavior.
Captured data will also be used for robot motion planning based on long-term prediction of human motions to ensure safe and efficient human-robot interaction. More details can be found in [1].

\section{References}

[1] H. Ding, G. Reissig, K. Wijaya, D. Bortot, K. Bengler, and O. Stursberg. Human Arm Motion Modeling and Long-Term Prediction for Safe and Efficient Human-Robot-Interaction. In IEEE International Conference on Robotics and Automation (ICRA) (2011), pp. 5875-5880.

[2] C. Gaudart. Conditions for maintaining ageing operators at work - a case study conducted at an automobile manufacturing plant, Applied Ergonomics (31) (2000), 453-462.

[3] N. Hanajima, Y. Ohta, H. Hikita und M. Yamashita. Investigation of impressions for approach motion of a mobile robot based on psychophysiological analysis, In Proc. of the 2005 IEEE International Workshop on Robot and Human Interactive Communication (2005), 79-84. IEEE, Nashville, TN, Aug, ISBN 0780392744.

[4] IEC 61508. Functional Safety of Electrical/Electronic/Programmable Electronic Safety-related Systems (2002).

[5] IEC 62508. Guidance on human aspects of dependability (2010).

[6] W. Karwowski, M. Rahimi, H. Parsaei, B. R. Amarnath und N. Pongpatanasuegsa. The effect of simulated accident on worker safety behavior around industrial robots, International Journal of Industrial Ergonomics (7) (1991), 229-239, ISSN 01698141 .

[7] M. Nagamachi and Y. Anayama. Ergonomic study of industrial robots (1) - The experiments of unsafe behaviour on robot manipulation (NIOSH Translation), Japanese Journal of Ergonomics (19) (1983), 259-264.

[8] NASA TLX Homepage. Retrieved from http://humansystems.arc.nasa.gov/groups/TLX/

[9] H. M. Parsons. Human factors in industrial robot safety, Journal of Occupational Accidents (8) (1986), 25-47, ISSN 03766349 .

[10]F. Schiller. The Relation between Safety and Reliability in Automation from the Safety Perspective. 11th Int. Symposium on System-Modelling-Control. SMC'2005 (2005). Zakopane. 13-19.

[11] S. Shibata und H. Inooka. "Psychological evaluations of robot motions, International Journal of Industrial Ergonomics (21) (1998), 483-494, ISSN 0169-8141.

[12]D. Stengel, B. Ostermann, H. Ding, D. Bortot, F. Schiller, O. Stursberg, K. Bengler, M. Huelke, F. Som, U. Strunz. An Approach for Safe and Efficient Human-Robot Collaboration, In $6^{\text {th }}$ International Conference Safety of Industrial Automated Systems (SIAS 2010) (2010), Tampere, ISBN 978-952-518340-5.

[13]N. Tejima. Psychological Safety of Rehabilitation Robots (1), Speed, Distance from Human Face to Robot Hand and Fear, In Proc. of the $10^{\text {th }}$ Annual Conference of Robotics Society of Japan (3) (1992), 1107-1108.

[14] Vicon Motion Systems Limited. Retrieved from http://www.vicon.com/

[15] Y. Yamada, Y. Umetani und Y. Hirasawa. Proposal of a psychophysiological experiment system applying the reaction of human pupillary dilation to frightening robot motions, In Proc. of the 1999 IEEE International Conference on Systems, Man, 
and Cybernetics (2) (1999), 1052-1057, IEEE, ISBN 0780357310 .

[16]T. Yamamoto, M. Jindai, S. Shibata und A. Shimizu. An avoidance planning of robots using fuzzy control considering human emotions, In IEEE SMC'99 Conference Proceedings (6) (2002), 976-981, IEEE, ISBN 0780357310 